Chirurg 2015 · 86:1070

DOI 10.1007/s00104-015-0094-9

Online publiziert: 13. Oktober 2015

(c) Springer-Verlag Berlin Heidelberg 2015

CrossMark

J. Reibetanz • C.T. Germer

Klinik für Allgemein-, Viszeral-, Gefäß- und Kinderchirurgie, Universitätsklinik Würzburg, Würzburg, Deutschland

\title{
Optimales Management des Morbus Crohn nach intestinaler Resektion
}

\section{Ergebnisse}

postoperativen Jahres erleiden bis zu $90 \%$ dieser Patienten ein endoskopisches und bis zu $30 \%$ ein klinisches Erkrankungsrezidiv, und $70 \%$ der Patienten müssen im weiteren Verlauf der Erkrankung erneute operiert werden. Das optimale Management zur postoperativen Rezidivprävention ist weiterhin kontrovers.

\section{Fragestellung und Methode}

Die randomisierte POstoperative Crohn's Endoscopic Recurrence (POCER) Studie analysierte unterschiedliche Managementstrategien zur Rezidivprophylaxe bei M. Crohn unter Berücksichtigung des individuellen Rezidivrisikos und des Benefits einer gezielten postoperativen Koloskopie. Ziel war die Identifikation der optimalen Nachsorgestrategie.

Hierzu wurden Patienten nach vorangegangener intestinaler Resektion (und endoskopisch einsehbarer Anastomose) 2:1 in unterschiedliche Therapiearme randomisiert: „active-care“ (Koloskopie nach 6 Monaten) vs. „standard-care“ (keine Koloskopie). Alle Patienten erhielten eine medikamentöse Rezidivprophylaxe (Metronidazol für 3 Monate, bei hohem Rezidivrisiko zusätzlich Thiopurine bzw. Adalimumab). Patienten der Active-care-
Gruppe erhielten 6 Monate postoperativ in Abhängigkeit des mukosalen Befalls eine Anpassung der medikamentöse Therapie („step-up“), bei Patienten der Standard-care-Gruppe wurde die postoperative Rezidivprophylaxe bis 18 Monate postoperativ unverändert fortgeführt.

Primärer Endpunkt der Studie war der Nachweis und Schweregrad eines endoskopischen Rezidivs 18 Monate postoperativ gemessen am Rutgeerts-Score.

\section{Diskussion und Fazit des Reviewers}

Die aktuelle Arbeit belegt eindrücklich, dass eine risikoadaptierte medikamentöse Rezidivprophylaxe zusammen mit einer 6-Monats-Endoskopie und hieran angepasster Step-up-Therapie der alleinigen (konventionellen) medikamentösen Therapie in der Vermeidung des postoperativen Crohn-Rezidivs signifikant überlegen ist. Der selektive Einsatz von potenten Immunsuppressiva auf Grundlage des früh-postoperativen Endoskopiebefundes scheint somit sinnvoller als ihre grundsätzliche Anwendung bei allen Patienten mit hohem Rezidivrisiko.

Im Gegensatz zur Empfehlung der deutschen Leitlinie zur Durchführung einer Ileokoloskopie (innerhalb des ersten Jahres nach in intestinaler Resektion) „falls sich aus dem endoskopischen Befund therapeutische Konsequenzen ergeben würden " [1] muss nach vorliegender Studie für eine routinemäßige Koloskopie plädiert werden.

\section{Korrespondenzadresse}

ser Patienten innerhalb des Folgejahres in Remission brachte. Im Gegenzug zeigten Active-care-Patienten, die sich bei der 6-Monats-Koloskopie in Remission befanden (und daher keine Step-up-Therapie erhielten), in $41 \%$ der Fälle innerhalb eines Jahres ein Erkrankungsrezidiv. Insgesamt fanden sich 18 Monate postoperativ mit $49 \%$ vs. $67 \%(p=0,03)$ endoskopische Rezidive signifikant seltener bei Patienten der Active-care-Gruppe als bei Patienten der Standard-care-Gruppe. Parallel dazu zeigten mehr Patienten der Active-care-Gruppe einen mukosalen Normalbefund als Standard-care-Patienten [22 vs. $8 \%(p=0,03)$ ].

\section{J. Reibetanz}

Klinik für Allgemein-, Viszeral-, Gefäß- und

Kinderchirurgie,

Universitätsklinik Würzburg

97080 Würzburg

reibetanz_j@ukw.de

Interessenkonflikt. J. Reibetanz und C.T. Germer geben an, dass kein Interessenkonflikt besteht.

\section{Literatur}

1. Preiß JC, Bokemeyer B, Buhr HJ et al (2014) Aktualisierte S3-Leitlinie. Diagnostik und Therapie des M. Crohn. Z Gastroenterol 52(12):1431-1484

\section{Einhaltung ethischer Richtlinien}

